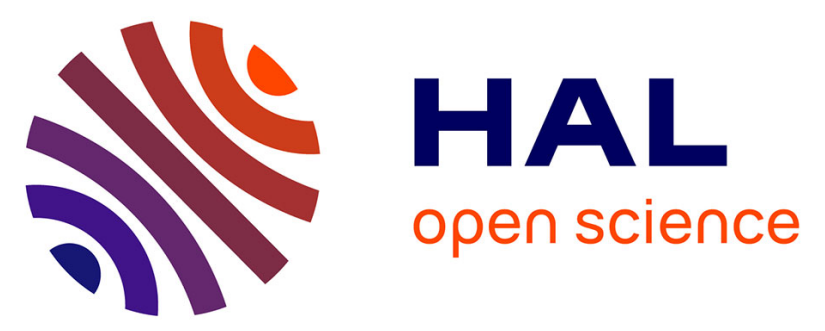

\title{
Comparison of predictive controllers for locomotion and balance recovery of quadruped robots
}

\author{
Thomas Corbères, Thomas Flayols, Pierre-Alexandre Léziart, Rohan \\ Budhiraja, Philippe Souères, Guilhem Saurel, Nicolas Mansard
}

\section{To cite this version:}

Thomas Corbères, Thomas Flayols, Pierre-Alexandre Léziart, Rohan Budhiraja, Philippe Souères, et al. Comparison of predictive controllers for locomotion and balance recovery of quadruped robots. IEEE International Conference on Robotics and Automation (ICRA 2021), May 2021, Xi'an, China. 10.1109/ICRA48506.2021.9560976 . hal-03034022v2

\section{HAL Id: hal-03034022 \\ https://hal.laas.fr/hal-03034022v2}

Submitted on 9 Sep 2021

HAL is a multi-disciplinary open access archive for the deposit and dissemination of scientific research documents, whether they are published or not. The documents may come from teaching and research institutions in France or abroad, or from public or private research centers.
L'archive ouverte pluridisciplinaire HAL, est destinée au dépôt et à la diffusion de documents scientifiques de niveau recherche, publiés ou non, émanant des établissements d'enseignement et de recherche français ou étrangers, des laboratoires publics ou privés. 


\title{
Comparison of predictive controllers for locomotion and balance recovery of quadruped robots
}

\author{
Thomas Corbères ${ }^{\mathrm{a}}$, Thomas Flayols ${ }^{\mathrm{a}, \mathrm{b}}$, Pierre-Alexandre Léziart ${ }^{\mathrm{a}}$, Rohan Budhiraja ${ }^{\mathrm{a}}$, \\ Philippe Souères ${ }^{\mathrm{a}}$, Guilhem Saurel ${ }^{\mathrm{a}}$, Nicolas Mansard ${ }^{\mathrm{a}, \mathrm{b}}$
}

\begin{abstract}
As locomotion decisions must be taken by considering the future, most existing quadruped controllers are based on a model predictive controller (MPC) with a reduced model of the dynamics to generate the motion and a wholebody controller to execute it. Yet the simplifying assumptions of the MPC are often chosen ad-hoc or by intuition. In this article, we focus on a set of MPCs and analyze the effect of chosen model reductions on the behavior of the robot. Based on existing formulations, we present additional controllers to better understand the influence of model reductions on the controller capabilities. Finally, we propose a robust predictive controller capable of optimizing the foot placements, gait period, centerof-mass trajectory and ground reaction forces. The behavior of these controllers is statistically evaluated in simulation. This empirical study aims to assess the relative importance of the components of the optimal control problem (variables, costs, dynamics) to be able to take reasoned decisions instead of arbitrarily emphasizing or neglecting some of them. We also provide a qualitative study in simulation and on the real robot Solo-12.
\end{abstract}

\section{INTRODUCTION}

Locomotion with quadruped robots is both challenging and rewarding for roboticists. With lighter feet than bipeds and more stability, quadrupeds can be used for highly dynamic motions and gaits, as already done with Cheetah [1], Anymal [2] or HyQ [3]. For this purpose, controllers that can generate real-time motion trajectories are highly sought. Among them, non-linear model predictive controllers (MPC) [4], [5] can provide real-time computations [3], reactive control [6], [7], and because of optimal control over a time horizon, can deal with disturbances and perturbations to the system [8].

Whole-body dynamics allow maximum utilization of our knowledge about the model of the robot [10]. However, nonlinearity and high dimensions are prohibitive with respect to computation times. The classical consequence is to use reduced dynamics of lower dimensions instead [11]-[13], very often with additional assumptions to further simplify the non-linearities of the problem. In such cases, the plan provided by the reduced model is followed by the lowlevel controller using inverse dynamics, e.g. in [6], [14]. Several reduced models can be considered to build a MPC for quadruped locomotion: table-cart [11] (or with foothold optimization [14], [15]), centroidal [16] (or with contact timings optimization [17]). For quadruped robots, a sound reduction is to approximate the angular momentum to the rotation of

\footnotetext{
a LAAS-CNRS, Université de Toulouse, CNRS, Toulouse, France

${ }^{b}$ Artificial and Natural Intelligence Toulouse Institute, France

This work has been supported by the MEMMO European Union project within the H2020 Program under Grant Agreement No. 780684.
}

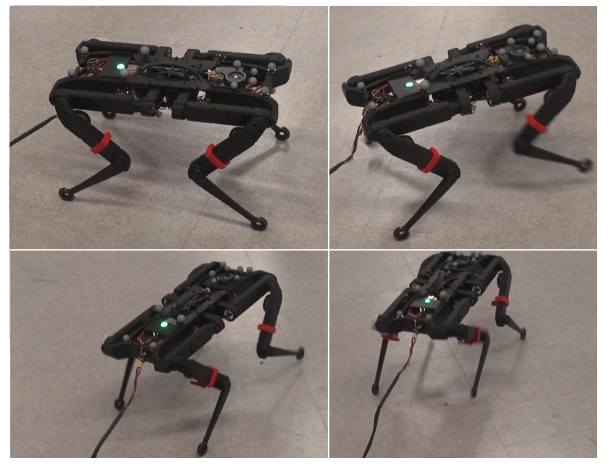

Fig. 1. Solo-12 [9] trotting during experiments. Motions from different controllers were tried on Solo. While all controllers were able to perform simple motions, we provide comparison of their performances for more extreme cases.

the rigid body and to neglect the limb dynamics [18]-[20], which we chose in this work [13].

The simplifications of dynamics are mostly the result of limited resources available for online real-time MPC. In this paper, we propose to simultaneously evaluate their advantages and drawbacks. After recalling the main concepts in Sec. II, we start by introducing the general optimal control problem (OCP) formulation. Then, based on the convex problem detailed in [13], the first OCP is presented in Sec. III to clearly define the optimization problem and serve as a reference for comparison. Three other optimal problems are then proposed by successively removing some linearization assumptions done in [13] (Sec. IV), extending the formulation to footholds optimization (Sec. V) and including the walk period as an optimization parameter (Sec VI). All OCPs are presented following the same template to better exhibit their similarities and particularities. As the two last OCP involve the simultaneous optimization of trajectories and static parameters (foothold positions and timings), we explain in Section VII how a differential dynamic programming (DDP) solver can be used to solve a parametric OCP. The MPC implementing the OCP to perform the feedback control of the robot is detailed in Sec. VIII and is used in Sec. IX to benchmark several OCP formulations both in simulation and on the robot Solo- 12 .

\section{Optimal Control With Reduced Dynamics}

The centroidal dynamics [21] describe the dynamics of the center of mass $(\mathrm{CoM})$ of the robot due to its interactions with the environment and corresponds to the under-actuated dynamics [22]. Since most quadruped robots are built with 
lightweight limbs, the centroidal dynamics described below are often used to approximate the whole-body dynamics :

$$
\begin{gathered}
m \ddot{p}=\sum_{i=1}^{n_{c}} f_{i}+m g \\
\mathcal{I} \dot{\omega}+\omega \times(\mathcal{I} \omega)=\sum_{i=1}^{n_{c}}\left(r_{i}-p\right) \times f_{i}
\end{gathered}
$$

where $p=(x, y, z)$ is the position of the body, $\omega$ is the angular velocity of the body, $m$ and $\mathcal{I}$ are mass and inertia of the body, and $g=(0,0,-9.8)$ is the gravity vector. $n_{c} 3 \mathrm{D}$ forces $f_{i}$ are applied at the contact points $r_{i}$. Consequently, the robot state is composed by linear and angular positions and velocities, belonging to $\mathbb{R}^{12}$ :

$$
x=\left[\begin{array}{llll}
p & \Theta & \dot{p} & \omega
\end{array}\right]^{T} .
$$

where $\Theta=(\theta, \phi, \psi)$ is the rotation of the body frame $\mathcal{R}_{l}$ with regards to the world frame $\mathcal{R}_{0}$. To facilitate the reading, we represent the rotation $\Theta$ by the 3 Euler angles. The rotation matrix from body to world is then: $R_{z y x}=$ $R_{z}(\psi) R_{y}(\phi) R_{x}(\theta)$.

In the next section, we will follow [13] and assume small body inclination $\phi$ and $\theta$. Consequently, i) the roll and pitch angles in the rotational matrices can be neglected: $R_{z}(\psi) R_{y}(\phi) R_{x}(\theta) \approx R_{z}(\psi)$. ii) $\omega=\dot{\Theta}$. iii) The representation of inertia matrix in world frame is simplified as: $\mathcal{I} \approx R_{z}(\psi) \mathcal{I}_{l} R_{z}(\psi)^{T}$ [23], and iv) the cross-product term $\omega \times(\mathcal{I} \omega)$ is neglected leading to a linear variation of the angular momentum:

$$
\frac{d}{d t}(\mathcal{I} \omega) \approx \mathcal{I} \dot{\omega}
$$

where $\mathcal{I}$ is approximately constant in the world frame. We can now set up the generic form of the optimal control problem for locomotion. The predicted time horizon can be divided using different contact phases. Each modification of ground contact - creation or deletion - is associated with a new phase $s$ entirely defined by its duration $T_{s}$ and the location of feet in contact $r_{s}$.

$$
\begin{aligned}
\min _{\{x\},\{u\}} & \sum_{s \in \text { phases }} \sum_{t=0}^{T_{s}} \ell\left(x_{t}, u_{t} \mid r_{s}\right)+\ell_{T}\left(x_{T}\right) \\
\text { s.t. } \quad & x_{0}=\hat{x} \\
& \forall t, s \quad x_{t+1}=f\left(x_{t}, u_{t} \mid r_{s}\right) \\
& \forall t \quad u_{t} \in \mathcal{U} \\
& \forall t, s \quad x_{t} \in \mathcal{X}_{s}
\end{aligned}
$$

where $\ell_{k}$ and $\ell_{N}$ are respectively the running and terminal cost. $\{x\}$ and $\{u\}$ are the decision variables which we take as discretized at the nodes indexed by $t$. The control vector $u$ contains the $3 \mathrm{D}$ forces at each contact point, constrained to the friction cone $\mathcal{U}$. The centroidal states $x$ should be chosen so that there exists a valid whole-body movement that can achieve $x$ [22]. We put this last constraint under the abstract form $x \in \mathcal{X}_{s}$ which depends on the phase s. This would take the form of kinematic constraints as described in Sec. IV-B. The OCP presented here is still quite abstract. However, in the following sections, it will be derived into several versions of increasing complexity, starting from the OCP formulated in [22]. Our goal is to show how several motion features can be formulated to improve the accuracy of the decisions the MPC is capable of and to experimentally show the consequences of these improvements.

\section{LINEARIZATION OF THE DYNAMICS}

The first problem is a simple reformulation of the convex formulation proposed in [13]. The CoM is approximated by the desired CoM $p^{*}$ in the Euler equation (1b). With this assumption, we can reduce the complexity and make the system convex as the dynamic matrix no longer contains coupling between control and state vector:

$$
\mathcal{I} \dot{\omega}=\sum_{i=1}^{n_{c}}\left(r_{i}-p\right) \times f_{i} \approx \sum_{i=1}^{n_{c}}\left(r_{i}-p^{*}\right) \times f_{i}
$$

\section{A. Convex Dynamic Model}

The chosen dynamics (1a) - (5) is discretized with an implicit scheme of integration such that $P_{t+1}=P_{t}+\Delta t V_{t+1}$ to estimate the position more accurately, bringing $\Delta t^{2}$ terms to the $B$ matrix.

$$
\begin{aligned}
x_{t+1} & =f_{t}\left(x_{t}, u_{t}\right)=A x_{t}+B u_{t} \\
A & =\left[\begin{array}{cc}
I_{6} & \Delta t I_{6} \\
0_{6} & I_{6}
\end{array}\right] \\
B & =\left[\begin{array}{ccc}
\frac{\Delta t^{2}}{m} I_{3} & \ldots & \frac{\Delta t^{2}}{m} I_{3} \\
\Delta t^{2} \mathcal{I}^{-1}\left[r_{1}-p *\right]_{\times} & \ldots & \Delta t^{2} \mathcal{I}^{-1}\left[r_{n}-p *\right]_{\times} \\
\frac{\Delta t}{m} I_{3} & \ldots & \frac{\Delta t}{m} I_{3} \\
\Delta t \mathcal{I}^{-1}\left[r_{1}-p *\right]_{\times} & \ldots & \Delta t \mathcal{I}^{-1}\left[r_{n}-p *\right]_{\times}
\end{array}\right]
\end{aligned}
$$

with $\Delta t$ the integration time between nodes, $I_{6}$ the identity matrix of size 6 and $[\ldots]_{\times}$a $3 \times 3$ skew-symmetric matrix representing cross products as matrix multiplications.

\section{B. Cost function}

The cost function consists of three cost terms. The first one is a quadratic cost which penalizes the error between the state vector and the desired state. The second quadratic cost penalizes the relative command vector. We use the relative reference force $f_{z}^{r e f}=f_{z}-\frac{m g}{n_{c}}$, where $f_{z}$ is the normal ground reaction force, since it brings the forces to equilibrium and also leads to a faster convergence. Finally, we use a penalty cost to implement the friction cone inequality constraints. Because the solver we use works by penalization, they cannot be formulated as such and instead 
are translated in a penalisation term using a discrete 4-facet cone approximation and for each foot:

$$
\begin{gathered}
\ell_{\text {cone }, i}(f)=\frac{1}{2}\left\|\left(f_{x}-\mu f_{z}\right)^{+}\right\|^{2}+\frac{1}{2}\left\|\left(-f_{x}-\mu f_{z}\right)^{+}\right\|^{2} \\
+\frac{1}{2}\left\|\left(f_{y}-\mu f_{z}\right)^{+}\right\|^{2}+\frac{1}{2}\left\|\left(-f_{y}-\mu f_{z}\right)^{+}\right\|^{2}+\frac{1}{2}\left\|\left(f_{z}\right)^{+}\right\|^{2}
\end{gathered}
$$

where $y^{+}=\max (y, 0)$. The cost is thus activated and strongly penalized only if $u$ does not respect the inequality constraints. However, this penalisation formulation does not guarantee that the constraints will be respected. Some margin for the coefficient friction is needed. We experimentally show that this approximation works very well and presents some results in Sec. IX-A.

\section{KEEPING THE BILINEAR DYNAMICS}

\section{A. Non Linear model}

In this second OCP, the assumption (5) on the lever arm is withdrawn. We no longer use the desired CoM position $p^{*}$ in the cross product but instead the predicted CoM $p$. The B matrix (6c) therefore varies with the state and the coupling between state and control is re-introduced.

\section{B. Kinematic constraint}

In the previous controller, no precautions are taken to ensure that $x$ is admissible (i.e. $x \in \mathcal{X}_{s}$ ). In particular it did not guarantee that the CoM would not move too far away from the contact. As we now accept to have a nonlinear formulation, we take that as an opportunity to add a term to enforce it. This kinematic limit is approximated by penalizing distances between shoulders and their associated contact point when over $80 \%$ of the leg limit. The position of a shoulder in $R_{0}$ can be formulated such as :

$$
\left\{\begin{array}{l}
x_{s h}=x+p_{x}-p_{y} \psi \\
y_{s h}=y+p_{y}+p_{x} \psi \\
z_{s h}=z+p_{y} \phi-p_{x} \theta
\end{array}\right.
$$

where $\left(\begin{array}{lll}p_{x} & p_{y} & 0\end{array}\right)^{T}$ is the position of the shoulder in $\mathcal{R}_{l}$ and $\left(\begin{array}{lll}x & y & z\end{array}\right)^{T}$ the position of the CoM in $\mathcal{R}_{0}$. The shoulder-to-cost penalization is thus the following :

$\ell_{s h}\left(x_{t}\right)=\left\|\left(\left(x_{s h}-x_{c}\right)^{2}+\left(y_{s h}-y_{c}\right)^{2}+\left(z_{s h}-z_{c}\right)^{2}-d_{l i m}^{2}\right)^{+}\right\|^{2}$ where $x_{c}=\left(x_{c}, y_{c}, z_{c}\right)^{T}$ is the contact placement in $R_{0}$.

\section{Simultaneous Foothold OPTIMIZATION}

The third controller additionally optimizes feet placements. The set of contact $\{r\}$, containing the contact locations $r_{s}$ for each phase $s$, are therefore a decision variable. This new OCP can be rewritten as:

$$
\begin{aligned}
\min _{\{x\},\{u\},\{r\}} & \sum_{s \in \text { phases }} \sum_{t=0}^{T_{s}} \ell\left(x_{t}, u_{t} \mid r_{s}\right)+\ell_{T}\left(x_{T}\right) \\
\text { s.t. } \quad & x_{0}=\hat{x} \\
& \forall t, s \quad x_{t+1}=f\left(x_{t}, u_{t} \mid r_{s}\right)
\end{aligned}
$$

In addition to the four cost terms described earlier in Sec. III and Sec. IV, namely the state regularization, the relative force regularization, the friction cone violation penalty and the kinematic limit penalty, three other quadratic costs are proposed to reduce the search space : i) The distance of the footstep is penalized. ii) The contact placement is penalised to keep the position of the foot around a certain heuristic. The same heuristic terms given to previous OCP models for contact points serve here to lead the optimization. iii) A last cost term is proposed which allows the optimization of the contact placement to be stopped when the foot is approaching the ground. It avoids destabilisation at the end of the flying phase by preventing lateral feet velocities just before touchdown. This last cost term disappears in the next controller.

\section{OCP WITH PERIOD OPTIMIZATION}

This last OCP formulation includes contact timings as a decision variable in addition to the decision variables of (9). Thus, we evaluate the behaviour of the OCP while optimizing the gait period, the footholds position, the trajectory of the CoM and the reaction forces at the same time :

$$
\begin{aligned}
\min _{\{x\},\{u\},\{r\},\{T\}} & \sum_{s \in \text { phases }} \sum_{t=0}^{T_{s}} \ell\left(x_{t}, u_{t} \mid r_{s}\right)+\ell_{T}\left(x_{T}\right) \\
\text { s.t. } \quad & x_{0}=\hat{x} \\
& \forall t, s \quad x_{t+1}=f\left(x_{t}, u_{t} \mid r_{s}\right)
\end{aligned}
$$

Looking only at the cendroidal dynamics, the solver will always take advantage of reducing the duration of the contact phase. A lower gait period means the CoM falls during a shorter duration until the next contact switch, hence a better stabilization capability. Yet, short duration of the contact phases implies high velocity of the flying feet, in particular if the solver also decides to take large steps. A cost term needs to be added to carry this information. Trajectories of the flying feet are represented by 5-th order polynomial functions chosen to nullify the speed and acceleration at touchdown and to take into account the current speed and acceleration of the flying feet. Only the lateral velocities are penalized since they are higher than the speed along the $\mathrm{Z}$ axis during the movement. The maximum speed along this trajectory is only a function of the start and end locations, and of the current velocity of the foot if any. For predicted phases that have not started yet, maximum speed is reached at the middle of the polynomials and thus easily evaluated. The maximum of a 4 th order polynomial function with non null initial conditions cannot be computed in a reasonable time. That is why the velocity of flying feet is evaluated multiple times along the trajectory at time $\alpha \Delta T$, where $\Delta T$ is the duration of the flying phase and $\alpha$ the percentage of trajectory travelled :

$$
V_{x}(\alpha \Delta T)=V_{0} b_{0}(\alpha)+\Delta \operatorname{Tacc}_{0} b_{2}(\alpha)+\frac{\Delta x}{\Delta T} b_{3}(\alpha)
$$

where $V_{x}$ is the speed of the foot along the $\mathrm{X}$ axis, $\Delta x$ is the distance traveled by the foot during the phase along the $\mathrm{X}$ axis, $v_{\text {lim }}$ the speed limit, $b$ constant coefficients depending 
on $\alpha, V_{0}$ the current speed of the foot and $a c c_{0}$ is the current acceleration. For one foot, the penalization is thus :

$$
\begin{array}{r}
\ell_{\text {speed }}(\Delta T, \Delta x, \Delta y)=\sum_{\alpha}\left[\left(\Delta T b_{1}+\Delta T^{2} b_{2}+\Delta x b_{3}\right)^{2}\right. \\
\left.+\left(\Delta T b_{1}+\Delta T^{2} b_{2}+\Delta y b_{3}\right)^{2}-\Delta T V_{\text {lim }}^{2}\right]^{+}
\end{array}
$$

For the next phases, the same expression with $\alpha=\frac{1}{2}$ holds.

\section{DIFFERENTIAL DYNAMIC PROGRAMMING SOLVER}

To solve the OCP formulations described above, the differential dynamic algorithm programming (DDP) is used from the library Crocoddyl [24], [25]. The discrete dynamics model is inserted into a multiple shooting problem. For the two first OCP, the implementation is quite straightforward since the state and the control vector do not change along the temporal horizon. OCPs proposed in (9) and (10) are actually parameterized OCPs and to handle them with DDP the state variables are augmented with the new decision variables.

\section{A. OCP with foothold optimization}

For the foothold OCP, with $r_{t}$ being the position of the foot in $\mathcal{R}_{0}: y_{t+1}=\left(x_{t}, r_{t}\right)$. A first shooting node is introduced to handle the dynamics (1a), (1b) with the forces as control vector. The second one is used to determine the next position of the feet and as the contact timing is predefined, this node is inserted before the modification of the contact point.

$$
\forall t \quad y_{t+1}=\left(\begin{array}{c}
f_{t}\left(x_{t}, u_{t}, p_{t}\right) \\
g_{t}\left(p_{t}, u_{t}\right)
\end{array}\right)
$$

where $f$ corresponds to the discrete dynamic model, and $\mathrm{g}$ is a function which allocates the new position of the feet when the contact switch occurs.

For the model representing the dynamics, $f$ is the same as the non linear OCP and feet positions are constant:

$$
g_{t}\left(p_{t}, u_{t}\right)=p_{t}
$$

Regarding the model inserted between the dynamics models, the control corresponds now to the distance between the previous and the new contact point. The size of the control thus depends on the number of modified foot placements. In this node, the state $x_{t}$ is constant $f\left(x_{t}, u_{t}, p_{t}\right)=x_{t}$ and here is the variation of the foot position:

$$
g_{t}\left(p_{t}, u_{t}\right)=p_{t}+u_{t}
$$

\section{B. OCP with period optimization}

The same method is used and the state is augmented with the integration time between the nodes : $\Delta t_{s}=\frac{T_{s}}{N_{s}}$ where $N_{s}$ is the number of nodes in phase $s$. A third type of node whose control is of size 1 and equal to the integration time is inserted before each flying phase and stance phase. By modifying the integration time between the nodes the period of the phase is thus modified. The cost proposed on the state

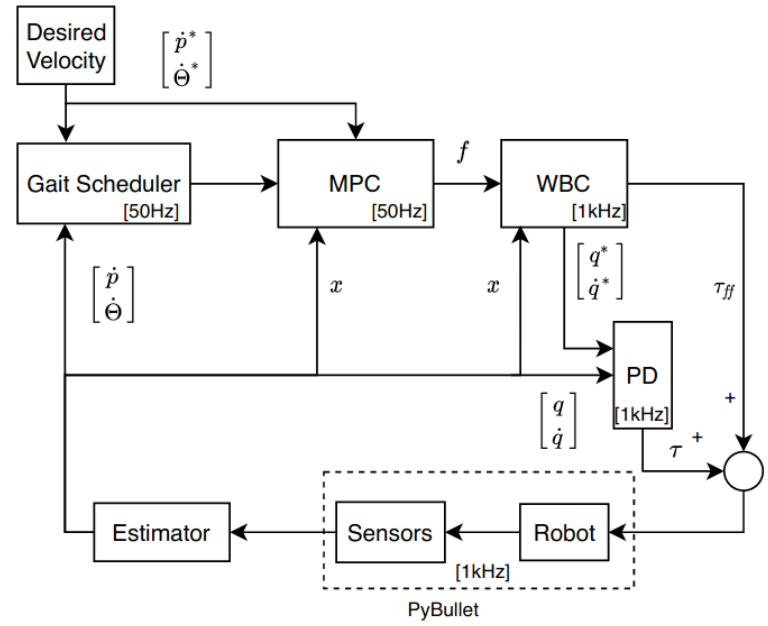

Fig. 2. Architecture of the controller. $x$ is the state vector, $q$ the joint configuration, $\tau$ the torque from the PD controller and $\tau_{f f}$ the feed-forward torques, $f$ the contact forces.

vector is no longer quadratic and depends on the integration time:

$$
\ell_{\text {state }}\left(x_{t} \mid s\right)=\frac{\Delta t_{s}}{2}\left(x_{t}-x^{*}\right)^{T} W_{s}\left(x_{t}-x^{*}\right)
$$

where $W_{s}$ the weight vector, $x_{t}$ the state and $x^{*}$ the desired state. Thus if no additional constraints are formulated the optimization solver tends to minimize the phase period by reducing the integration time to minimize the cost. This phenomena is highly sought since first it avoids falling into a local optimum. Then, this allows the optimization to reach a solution faster. To finish, we assume that the behaviour of the robot is more stable with a small gait period since the distance between footholds is lower and the quadrupedal support are more frequent. Therefore, a minimal integration time is required.

\section{IMPLEMENTATION DETAILS}

The four OCP formulations described above are tested, evaluated and compared in simulation as a model predictive controller with the Solo12 robot. They are integrated inside a state-of-the-art control architecture for cyclic quadrupedal locomotion as described in Fig. 2. It is close to the hybrid architecture proposed in [26] in which control blocks are explained in more details. The MPC block, running at $50 \mathrm{~Hz}$, is coupled with a whole body controller at $1 \mathrm{kHz}$. From the desired speed and walking pattern, the gait scheduler block outputs the heuristic to choose the footstep location and the sequence of contact phases. This heuristic is the same than described in [1]. The environment and the simulated feedback of sensors are generated using the PyBullet simulator [27]. We have chosen to run our test in simulation as each controller will be extensively tested until it reaches its limit, which we could not afford to do on the real robot.

\section{A. Model predictive controller}

The OCP formulations are tested in the MPC block. It receives the estimated state, the desired state resulting from 

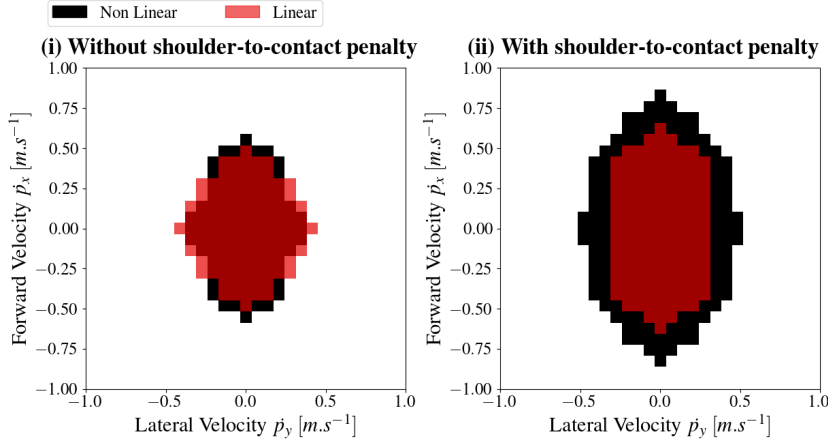

Fig. 3. Viable Operating Region. Comparison of linear and non linear MPC with the shoulder-to-contact point penalization on the right (ii). On the left (i), the non-linear solver tends to elevate the body, that the WBC then fails to track, although both solves behave quite similar. The benefits of completely modeling the lever arm dynamics is highlighted by the larger area on the right.

the reference velocity and feet positions in local frame given by the gait scheduler [1]. The MPC outputs ground reaction forces to apply at contact points and the predicted state evolution if they are applied. One period of gait is chosen for the temporal horizon, around $0.5 \mathrm{~s}$. The number of nodes is chosen depending on the integration time between them. 15 to 30 nodes are usually taken. To warm-start the MPC, the predicted state and calculated forces from the previous control cycle are slipped of one iteration into the timeline for the new control cycle. For the last node, the values at the equilibrium point are chosen.

\section{B. Whole body controller (WBC)}

Feet in swing phase follow a 5-th order polynomial trajectory in $\mathrm{X}, \mathrm{Y}$, and $\mathrm{Z}$ axis to ensure negative acceleration and velocity at the impact point. The trajectory is updated at each iteration with current acceleration/velocities of the foot and the new contact point. To avoid lateral velocities at touchdown, the contact point location is locked when $90 \%$ of the flying phase is done.

To generate the inverse dynamic control, we use a task space inverse dynamics (TSID) [28]. Using the estimated state, contact forces from the MPC and feet positions, the WBC computes the torques, position and velocities of joints. The computed torques are then tracked using a proportional derivative controller with feed-forward. In order to highlight the behavior of the MPC rather than the WBC, we voluntarily choose a high frequency for it at $1 \mathrm{Khz}$.

\section{RESULTS}

The main results obtained in simulation are presented in this section. To understand the difference in behavior induced by changes in OCPs, a statistical approach has been set up. The goal is to determine their respective viable operating region. A video showing some of our results can be found online $^{1}$.

1https: //peertube. laas.fr/videos/watch/ d6d90690-3262-4e61-9d03-08a2623726e3

\section{A. Checking the force-cone penalization}

To first validate the DDP solver, the initial linear controller was compared to the QP formulation proposed in [13], solved with the OSQP solver [29]. We checked by an empirical validation that 3 iterations of DDP solver were enough to solve the problem in every situation. We chose 16 nodes and a time horizon of $0.32 \mathrm{~s}$. On a forward velocity $V^{*}=$ $0.3 \mathrm{~m} \cdot \mathrm{s}^{-1}$, the root means square error (RMSE) between the forces computed by [13] and the forces computed by DDP solver using the force cone penalization was only $\Delta f=0.3 N$. The next results concerning the computation time were obtained with 5000 trials for one iteration of the DDP algorithm.

TABLE I

BENCHMARK OF THE DDP FOR OCPS

\begin{tabular}{|l|c|c|}
\hline OCP & Mean [ms] & Min - Max [ms] \\
\hline Linear [ms] & 0.327 & $0.302-0.882$ \\
\hline Non Linear [ms] & 0.351 & $0.305-0.938$ \\
\hline Footstep [ms] & 0.676 & $0.618-1.271$ \\
\hline Dt Optim [ms] & 0.769 & $0.725-1.511$ \\
\hline
\end{tabular}

\section{B. Influence of the lever arm assumption}

We then compared the formulations by running a complete MPC simulation with a given reference basis velocity. The simulation is validated if the robot reaches a steady cycle with the commanded velocity, and invalidated if the robot falls or does not reach it. We then plot the viable operating region $V_{x}-V_{y}$ and $V_{x}-\omega$. We first compare the linear OCP in section III with the non-linear OCP of section IV. In Fig. 3 on the right, we compare the two MPCs with the cost term introduced in section IV that penalizes the distance between the shoulder and the contact point. Without the kinematic constraint, convex and non-linear MPCs are quite equivalent. The areas are almost similar in Fig. 3-left. The non-linear MPC slightly improves the behaviour for forward velocities whereas it is less efficient for lateral velocities. Indeed, it takes advantage of the cross-product relative to the lever arm (1b) by elevating the robot body for such lateral velocities. It results in movements not achievable by the robot with the CoM too high.

The shoulder-to-contact penalty cost allows the OCP to better handle this issue. On the contrary, this term degrades the behaviour of the linear MPC for lateral velocities as highlighted by the Fig.3-right. The origin of this effect has not been identified yet. In general the non-linear OCP performs much better in particular at high speed. This non-linear problem does not need a complex warm-start to converge toward an acceptable solution. Both OCPs mentioned above requires the same number of iterations to converge and their computation time is equivalent.

\section{Optimization of the footsteps}

Fig. 4 summarizes the performances of the first three OCPs with shoulder-to-contact penalty. The non linear model with optimization of the footholds improves the robot walking 

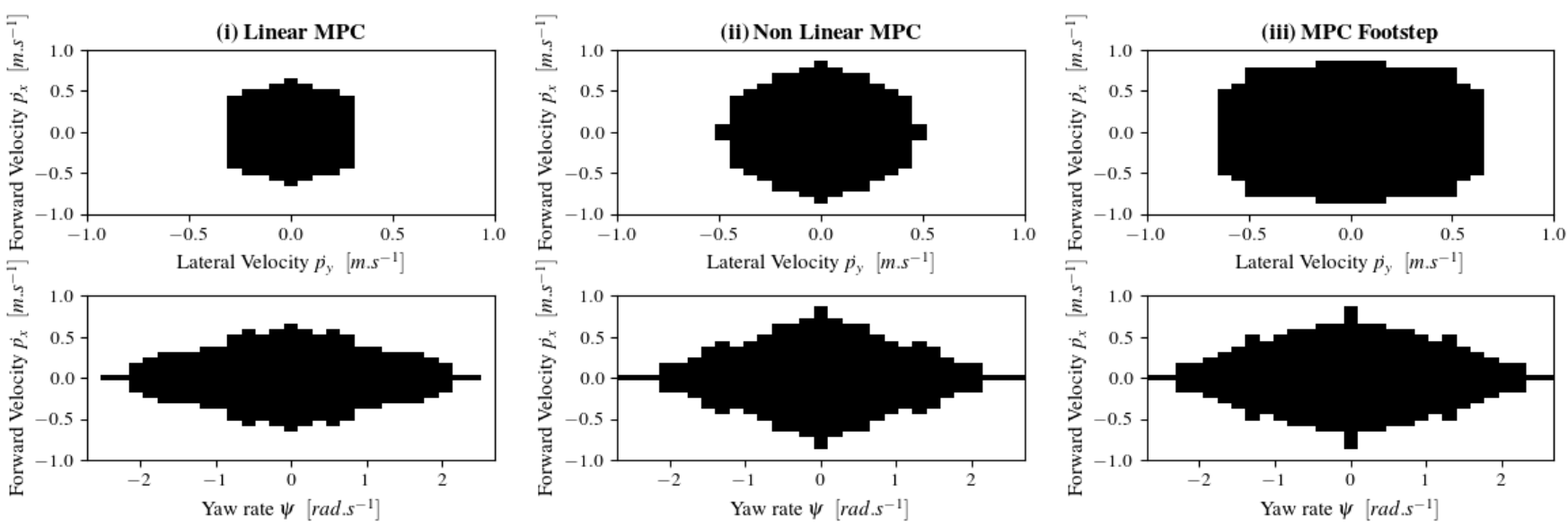

Fig. 4. Comparing the 3 OCP : (i) linear OCP, (ii) non-linear OCP with fixed footholds, (iii) non-linear OCP with foothold as decision variable. In the three cases, the shoulder-to-cost penalty is activated. Optimally deciding the footholds greatly helps to stabilize the system.

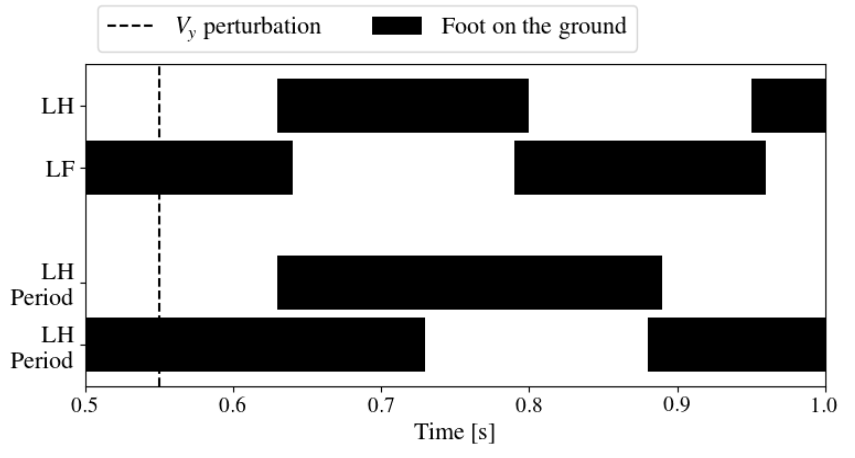

Fig. 5. Stance phase in dark for the left hind -LH- and front foot -LF-, depending on which controller is used (optimization of the period or not). The gait, predefined, is symmetric for the right feet.

behaviour by producing a more stable locomotion. However, the computation time is higher: one iteration of the DDP solver takes approximately $0.6 \mathrm{~ms}$. It also requires more iterations, up to 5 with a proper warm-start.

\section{Optimization of the gait period}

In nominal situation, the MPC tends to minimize the period as explained in VII-B. For a nominal walk, the timings are marginally changed by the solver and the viable operating region is not modified, even at high speed. Optimizing the gait period provides the most interesting results when the robot has to handle a perturbation. When a perturbation occurs, the controller is able to modify the duration of the flying phase and the duration of the stance phase. We have tried to quantify this augmented robustness, yet in simulation, all controllers resist unrealistic perturbations (up to $\left.\Delta_{V}=1 m \cdot s^{-1}\right)$. As an example, the system is perturbed by a linear velocity during a double support phase in Fig. 6. The stance phase is then extended as shown in Fig. 5 to allow a better rejection of the perturbation on the roll, pitch and yaw angles since it is more stable during quadrupedal support. A further disturbance analysis to properly quantify the controller stability should be incorporated in a more advanced version of the paper.
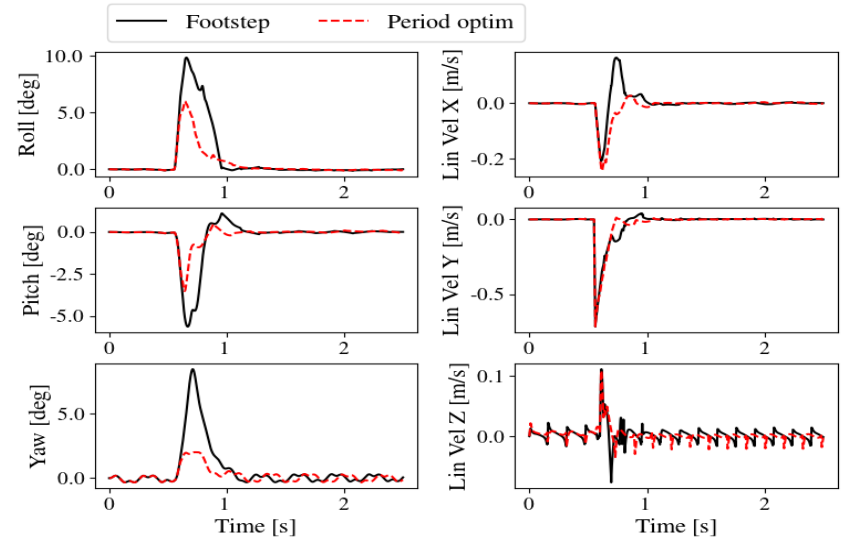

Fig. 6. Angular positions and linear velocities of the robot CoM in the world frame. Perturbation of the lateral velocity $-\mathrm{Y}$ axis- of $0.7 \mathrm{~m} . \mathrm{s}^{-1}$ during a double contact phase.

\section{Conclusion and Perspective}

We have proposed several formulations of MPCs for quadruped locomotion and evaluated statistically and qualitatively the impact of their formulation on the control.

Our first observation is that the linearity of either the dynamics or the cost is not a mandatory feature for computational efficiency. Actually, we would be more interested by strict convexity, for which linearity is only a proxy [30].

We have empirically demonstrated the importance of considering the exact dynamics of the lever arm in the MPC, the kinematic limit, the positions and timing of the contacts. We showed that these non-linear features only marginally increase the computational burden. On the other hand, some approximations of the dynamics, or some constraints that are discarded from the problem because of their non-linearity, have a strong impact on the quality of the controller. We believed the same comparative methodology should be continued and could integrate other missing terms next. We are also looking at a way to extend the systematic validation on real hardware without damaging the robot. 


\section{REFERENCES}

[1] D. Kim, J. D. Carlo, B. Katz, G. Bledt, and S. Kim, "Highly dynamic quadruped locomotion via whole-body impulse control and model predictive control," 2019.

[2] M. Hutter, C. Gehring, D. Jud, A. Lauber, C. D. Bellicoso, V. Tsounis, J. Hwangbo, K. Bodie, P. Fankhauser, M. Bloesch, R. Diethelm, S. Bachmann, A. Melzer, and M. Hoepflinger, "Anymal - a highly mobile and dynamic quadrupedal robot," in 2016 IEEE/RSJ International Conference on Intelligent Robots and Systems (IROS), 2016, pp. 38-44.

[3] M. Neunert, M. Stauble, M. Giftthaler, C. D. Bellicoso, J. Carius, C. Gehring, M. Hutter, and J. Buchli, "Whole-Body Nonlinear Model Predictive Control Through Contacts for Quadrupeds," IEEE Robotics and Automation Letters, vol. 3, no. 3, pp. 1458-1465, jul 2018. [Online]. Available: http://ieeexplore.ieee.org/document/8276298/

[4] E. Todorov, T. Erez, and Y. Tassa, "MuJoCo: A physics engine for model-based control," in 2012 IEEE/RSJ International Conference on Intelligent Robots and Systems. IEEE, oct 2012, pp. 5026-5033. [Online]. Available: http://ieeexplore.ieee.org/document/6386109/

[5] J. Koenemann, A. Del Prete, Y. Tassa, E. Todorov, O. Stasse, M. Bennewitz, and N. Mansard, "Whole-body model-predictive control applied to the HRP-2 humanoid," in IEEE International Conference on Intelligent Robots and Systems, vol. 2015-Decem. IEEE, sep 2015, pp. 3346-3351. [Online]. Available: http://ieeexplore. ieee.org/document/7353843/

[6] C. Dario Bellicoso, F. Jenelten, P. Fankhauser, C. Gehring, J. Hwangbo, and M. Hutter, "Dynamic locomotion and whole-body control for quadrupedal robots," in 2017 IEEE/RSJ International Conference on Intelligent Robots and Systems (IROS). IEEE, sep 2017, pp. 3359-3365. [Online]. Available: http://ieeexplore.ieee.org/ document/8206174/

[7] M. V. Minniti, F. Farshidian, R. Grandia, and M. Hutter, "Whole-body mpc for a dynamically stable mobile manipulator," IEEE Robotics and Automation Letters, vol. 4, no. 4, pp. 3687-3694, 2019.

[8] H. Kim, H. Seo, S. Choi, C. J. Tomlin, and H. J. Kim, "Incorporating Safety Into Parametric Dynamic Movement Primitives," IEEE Robotics and Automation Letters, vol. 4, no. 3, pp. 2260-2267, jul 2019. [Online]. Available: https://ieeexplore.iee.org/document/8648156/

[9] F. Grimminger, A. Meduri, M. Khadiv, J. Viereck, M. Wüthrich, M. Naveau, V. Berenz, S. Heim, F. Widmaier, T. Flayols, J. Fiene, A. Badri-Spröwitz, and L. Righetti, "An open torque-controlled modular robot architecture for legged locomotion research," IEEE Robotics and Automation Letters, vol. 5, no. 2, pp. 3650-3657, 2020.

[10] M. Neunert, F. Farshidian, A. W. Winkler, and J. Buchli, "Trajectory Optimization Through Contacts and Automatic Gait Discovery for Quadrupeds," IEEE Robotics and Automation Letters, vol. 2, no. 3, pp. 1502-1509, jul 2017. [Online]. Available: http://ieeexplore.iee.org/document/7845678/

[11] S. Kajita, F. Kanehiro, K. Kaneko, K. Fujiwara, K. Harada, K. Yokoi, and H. Hirukawa, "Biped walking pattern generation by using preview control of zero-moment point," in IEEE International Conference on Robotics and Automation. IEEE, 2003, pp. 1620-1626. [Online]. Available: http://ieeexplore.ieee.org/document/1241826/

[12] A. W. Winkler, F. Farshidian, D. Pardo, M. Neunert, and J. Buchli, "Fast Trajectory Optimization for Legged Robots Using VertexBased ZMP Constraints," IEEE Robotics and Automation Letters, vol. 2, no. 4, pp. 2201-2208, oct 2017. [Online]. Available: http://ieeexplore.ieee.org/document/7970144/

[13] J. Di Carlo, P. M. Wensing, B. Katz, G. Bledt, and S. Kim, "Dynamic Locomotion in the MIT Cheetah 3 Through Convex Model-Predictive Control," in 2018 IEEE/RSJ International Conference on Intelligent Robots and Systems (IROS). IEEE, oct 2018, pp. 1-9. [Online]. Available: https://ieeexplore.iee.org/document/8594448/

[14] A. W. Winkler, F. Farshidian, M. Neunert, D. Pardo, and J. Buchli, "Online walking motion and foothold optimization for quadruped locomotion," in 2017 IEEE International Conference on Robotics and Automation (ICRA), 2017, pp. 5308-5313.

[15] A. Herdt, H. Diedam, P.-B. Wieber, D. Dimitrov, K. Mombaur, and M. Diehl, "Online Walking Motion Generation with Automatic Footstep Placement," Advanced Robotics, vol. 24, no. 5-6, pp. 719-737, jan 2010. [Online]. Available: https://www.tandfonline.com/ doi/full/10.1163/016918610X493552

[16] B. Ponton, A. Herzog, S. Schaal, and L. Righetti, "A convex model of humanoid momentum dynamics for multi-contact motion generation," in 2016 IEEE-RAS 16th International Conference on
Humanoid Robots (Humanoids). IEEE, nov 2016, pp. 842-849. [Online]. Available: http://ieeexplore.ieee.org/document/7803371/

[17] J. Carpentier and N. Mansard, "Multicontact Locomotion of Legged Robots," IEEE Transactions on Robotics, vol. 34, no. 6, pp. 1441-1460, dec 2018. [Online]. Available: https://ieeexplore.ieee.org/ document $/ 8558661 / \mathrm{https} / /$ hal.archives-ouvertes.fr/hal-01520248/

[18] I. Mordatch, E. Todorov, and Z. Popović, "Discovery of complex behaviors through contact-invariant optimization," ACM Transactions on Graphics, vol. 31, no. 4, pp. 1-8, jul 2012. [Online]. Available: http://dl.acm.org/citation.cfm?doid=2185520.2185539

[19] A. W. Winkler, C. D. Bellicoso, M. Hutter, and J. Buchli, "Gait and Trajectory Optimization for Legged Systems Through Phase-Based End-Effector Parameterization," IEEE Robotics and Automation Letters, vol. 3, no. 3, pp. 1560-1567, jul 2018. [Online]. Available: http://ieeexplore.ieee.org/document/8283570/

[20] J. Di Carlo, P. M. Wensing, B. Katz, G. Bledt, and S. Kim, "Dynamic locomotion in the mit cheetah 3 through convex model-predictive control," in 2018 IEEE/RSJ International Conference on Intelligent Robots and Systems (IROS). IEEE, 2018, pp. 1-9.

[21] D. E. Orin, A. Goswami, and S.-H. Lee, "Centroidal dynamics of a humanoid robot," Autonomous Robots, vol. 35, no. 2-3, pp. 161-176, oct 2013. [Online]. Available: http://link.springer.com/10 1007/s10514-013-9341-4

[22] J. Carpentier, R. Budhiraja, and N. Mansard, "Learning Feasibility Constraints for Multicontact Locomotion of Legged Robots," in Robotics: Science and Systems XIII. Robotics: Science and Systems Foundation, jul 2017. [Online]. Available: http://www. roboticsproceedings.org/rss13/p31.pdf

[23] R. Featherstone, Rigid Body Dynamics Algorithms. Boston, MA Springer US, 2008. [Online]. Available: http://link.springer.com/10. 1007/978-0-387-74315-8

[24] C. Mastalli, R. Budhiraja, W. Merkt, G. Saurel, B. Hammoud, M. Naveau, J. Carpentier, S. Vijayakumar, and N. Mansard, "Crocoddyl: An Efficient and Versatile Framework for MultiContact Optimal Control," in ICRA 2020 - IEEE International Conference on Robotics and Automation, 2020. [Online]. Available: http://arxiv.org/abs/1909.04947

[25] J. Carpentier, G. Saurel, G. Buondonno, J. Mirabel, F. Lamiraux, O. Stasse, and N. Mansard, "The Pinocchio C++ library - A fast and flexible implementation of rigid body dynamics algorithms and their analytical derivatives," in SII 2019 - International Symposium on System Integrations, Paris, France, jan 2019. [Online]. Available: https://hal.laas.fr/hal-01866228

[26] P.-A. Léziart, T. Flayols, F. Grimminger, N. Mansard, and P. Souères, "Implementation of a Reactive Walking Controller for the New Open-Hardware Quadruped Solo-12," in 2021 IEEE International Conference on Robotics and Automation - ICRA, Xi'an, China, May 2021. [Online]. Available: https://hal.laas.fr/hal-03052451

[27] E. Coumans and Y. Bai, "Pybullet, a python module for physics simulation for games, robotics and machine learning," http://pybullet.org, 2016-2019.

[28] A. Del Prete, N. Mansard, O. E. Ramos, O. Stasse, and F. Nori, "Implementing Torque Control with High-Ratio Gear Boxes and Without Joint-Torque Sensors," International Journal of Humanoid Robotics, vol. 13, no. 01, p. 1550044, mar 2016. [Online]. Available: https://www.worldscientific.com/doi/abs/10.1142/ S0219843615500449

[29] B. Stellato, G. Banjac, P. Goulart, A. Bemporad, and S. Boyd, "OSQP: an operator splitting solver for quadratic programs," Mathematical Programming Computation, vol. 12, no. 4, pp. 637 672, dec 2020. [Online]. Available: http://link.springer.com/10.1007/ s12532-020-00179-2

[30] S. P. Boyd and L. Vandenberghe, Convex optimization. Cambridge University Press, 2004. [Online]. Available: https://dl.acm.org/citation. $\mathrm{cfm}$ ?id=993483 